\title{
77. The Treatment of Intracranial Aneurysm
}

\author{
Goro Tsuchiya, Shigeo Toya, Motohiro Tanaka, \\ Mitsuo Hattori, Toshiro Shizawa, \\ and Shigetomi MiYaKAWA \\ Division of Neurological Surgery, Keio University School of Medicine
}

We have experienced 41 aneurysms out of 36 patients in the last 3 years. The location of the aneurysms are as follow: 17 for the internal carotid, 12 for the anterior communicating artery, 3 for the anterior cerebral artery, 9 for the middle cerebral artery and 2 for the posterior cerebral artery.

The multiple aneurysms are over $10 \%$ of the total aneurysms of which most authors have reported. Only one case of the multiple aneurysms a re located on the bilateral carotid arteries and the rest of them are located within one hemisphere.

As for the indications of the treatment, as soon as the patient's condition recoveres, possibly between 10 days to 2 weeks after the episode, the angiogram should be performed and the surgery should be considered if it is indicated. As our new technique, Biobond is applied over the aneurysm and the clip, which is applied successfully, for the prevention of the falling and the securing of the clip.

The surgical indication of the multiple aneurysm is very difficult. We considered to attack the aneurysms in one stage whenever it is possible. The vasospasms, irregular shape of the aneurysm and the clinical findings are strongly suggested to the indication of the ruptured aneurysm. Which aneurysm should be attacked first, if the non-ruptured aneurysm is located more laterally then the ruptured one? We could operate only one case out of six multiple aneurysm cases. 